\title{
The pancreas: newer radiological methods of investigation
}

\author{
LOUIS KREEL \\ M.R.C.P., F.F.R. \\ Consultant Radiologist, \\ The Roval Free Hospital, London, W.C.1
}

THE visualization of the pancreas has not, as yet, become a routine procedure. The radiological diagnosis of pancreatic disease still depends, by and large, on indirect signs, whether these are shown on plain films or on barium examinations. The barium meal with special reference to the duodenal loop remains the sheet anchor of the average radiologist. The method and the signs of this examination have been considered in great detail by many authors, and are included in standard texts on the subject, whether radiological or clinical. Its accuracy is such as to leave much to be desired. It is estimated that only $20 \%$ of lesions of the head of the pancreas can be detected by this means. However, if the treatment of pancreatic diseases, especially neoplastic conditions, is to become effective, their diagnosis will have to be considerably earlier than is at present possible on the routine barium meal.

The diagnostic procedures to be considered are summarized in Table 1. Most of these procedures are at present only used in special centres, but as the value of these procedures will become more widely appreciated, so their use will spread. Some of these procedures will, in the near future, become part and parcel of the average radiologist's armamentarium. This, to a large measure, will depend on the desire to achieve accurate and early pre-operative diagnosis, both by the surgeon and radiologist.

\section{Duodenography}

The appearance of the duodenal loop, as seen on the routine barium meal, depends, among other things, on the pattern of the mucosal folds, the coating properties of acid-barium mixture leaving the stomach, inherent duodenal movements and the impression of the adjacent pancreas. This pattern often varies from film to film as the duodeum contracts and relaxes, and the lumen fills and empties as the barium passes through the duodenal loop. Often with a high gastric acidity there is a coarse mucosal pattern which makes interpretation

TABLE 1

\begin{tabular}{|c|c|c|c|}
\hline & Radiological procedure & Area of pancreas examined & Particular value \\
\hline A & $\begin{array}{l}\text { Duodenography } \\
\text { (i) Hypotonic } \\
\text { (ii) Gas distension double contrast }\end{array}$ & Head of pancreas & $\begin{array}{l}\text { Pancreatitis } \\
\text { Carcinoma } \\
\text { Diverticula of duodenum }\end{array}$ \\
\hline \multirow[t]{2}{*}{ B } & Pre-operative percutaneous cholangiography & Head of pancreas & $\begin{array}{l}\text { Extra-hepatic biliary obstruction } \\
\text { Carcinoma head of pancreas }\end{array}$ \\
\hline & Pre-operative duodenography combined & Head of pancreas & Carcinoma head of pancreas \\
\hline $\mathrm{C}$ & $\begin{array}{l}\text { Pancreatography }- \text { retroperitoneal gas } \\
\text { insufflation }+ \text { secretin }+ \text { intravenous } \\
\text { contrast medium and tomography }\end{array}$ & Body and tail & Tumours of pancreas \\
\hline \multirow[t]{2}{*}{ D } & $\begin{array}{l}\text { Arteriography }+ \text { secretin } \\
\text { (i) Coeliac axis } \\
\text { (ii) Superior mesenteric } \\
\text { (iii) Combined simultaneous coeliac axis } \\
\text { and superior mesenteric } \\
\text { (iv) Subselective pancreatic arteriography }\end{array}$ & $\begin{array}{l}\text { Whole pancreas but especially } \\
\text { head }\end{array}$ & $\begin{array}{l}\text { Carcinoma } \\
\text { Insulinoma }\end{array}$ \\
\hline & $\begin{array}{l}\text { Combined retroperitoneal air insufflation } \\
\text { and arteriography with intravenous } \\
\text { secretin and tomography }\end{array}$ & Whole pancreas & $\begin{array}{l}\text { Carcinoma } \\
\text { Insulinoma }\end{array}$ \\
\hline \multirow[t]{2}{*}{$\mathbf{E}$} & Operative pancreatography & Pancreatic ducts & Chronic pancreatitis \\
\hline & $\begin{array}{l}\text { Peroral intubation of sphincter of Oddi and } \\
\text { contrast injection }\end{array}$ & Head of pancreas & $\begin{array}{l}\text { Pancreatitis } \\
\text { Carcinoma }\end{array}$ \\
\hline $\mathbf{F}$ & Magna Scan with seleno-methionine & Whole pancreas & $\begin{array}{l}\text { Pancreatitis } \\
\text { Tumours }\end{array}$ \\
\hline
\end{tabular}




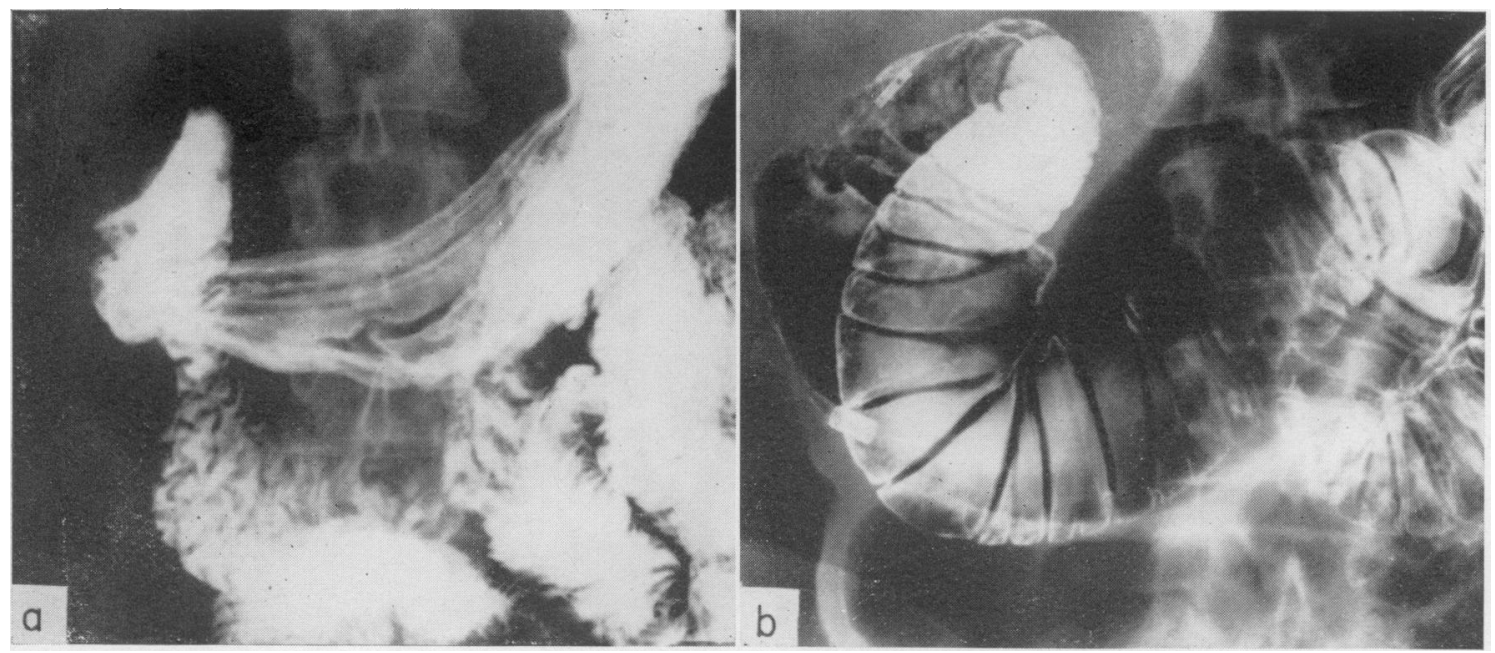

Fig. 1. The normal duodenal mucosal pattern on a routine barium meal (a) is compared with that obtained on gas distension duodenography in the same patient (b). In the routine barium meal the stomach and duodenal bulb as well as the jejunum are seen to overlie parts of the duodenal loop. The pattern in gas distension duodenography is quite different. Only transverse folds are visible running from margin to margin. The profile view of the mucosa shows that it is convex between the transverse folds.
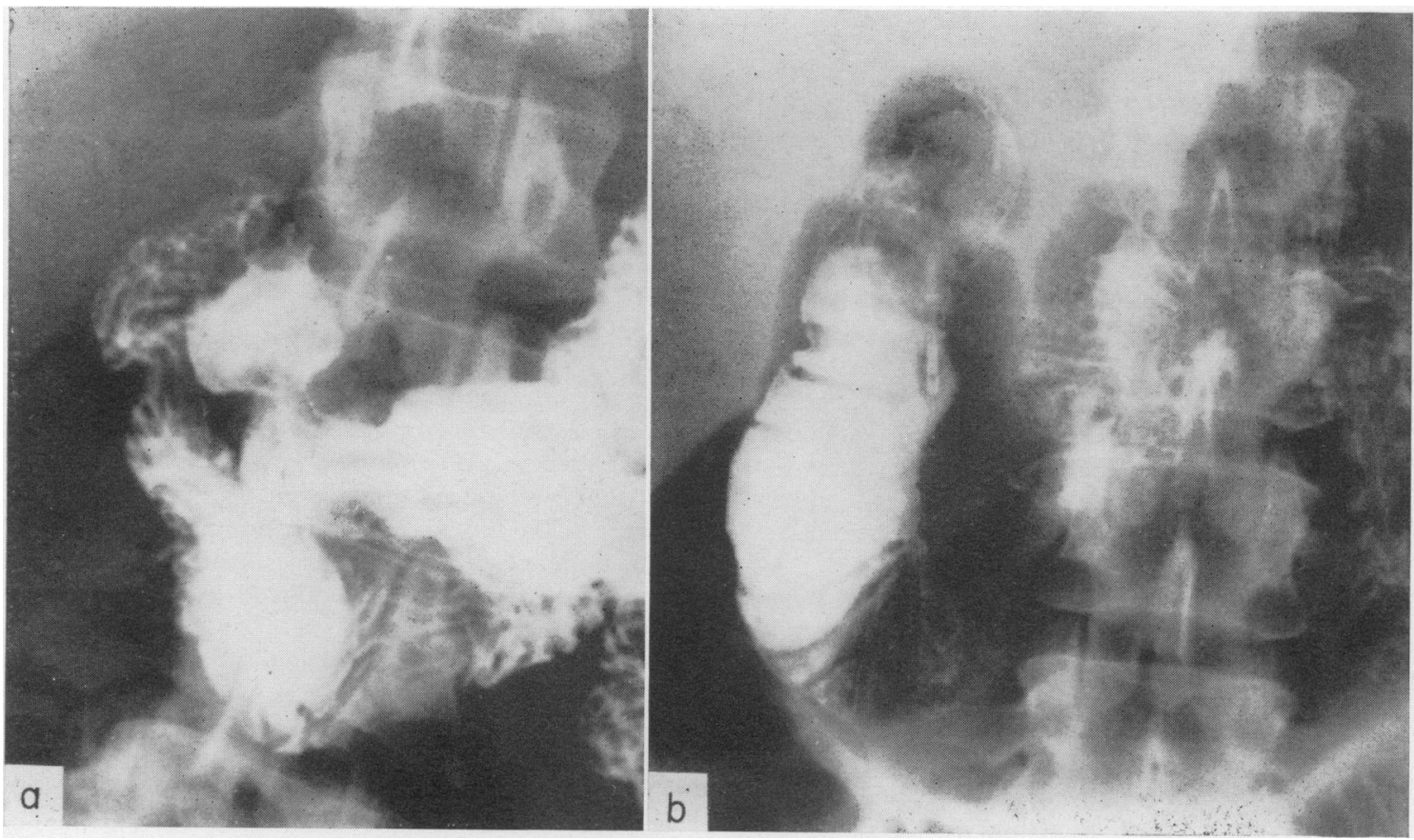

FIG. 2. The duodenal pattern on routine barium meal (a) is compared with that obtained on gas distension duodenography (b) in a case of chronic pancreatitis. The routine examination showed no abnormality, whereas on gas distension duodenography the appearances are obviously abnormal. The transverse folds are no longer visible in the duodenal wall adjacent to the pancreas, the inner margin has the 'inverted 3, shape, there is loss of the convexities between folds and an irregular fine striated pattern on this margin. 
of intrinsic duodenal abnormalities or changes due to pathology in the pancreas extremely difficult.

To overcome inherent movements, variable mucosal patterns and changes due to alterations in the barium, double-contrast gas-distension duodenography has been used. This technique is essentially simple to perform and can be carried out rapidly, with the minimum of discomfort to patients, without any special equipment. It is helpful to have television monitoring, but by no means essential.

\section{Method}

A quarter-of-an-hour before the procedure starts, the patient is given an amethocaine tablet to suck. The patient sits upright in a chair, and after a few puffs of $4 \%$ xylocaine on to the back of the throat, a double lumen gastro-duodenal tube (Scott-Harden) is passed. The patient is then placed in front of the screening table, and when the outer tube reaches the fundus of the stomach, the inner tube is passed on for about 4 in. Both tubes can then be pushed on together to follow the line of the greater curve. A mixture of $16 \mathrm{ml}$ Micropaque and $4 \mathrm{ml}$ water is then injected down the inner tube to enhance its visibility, but no barium is allowed to spill over into the stomach. The inner tube can then be guided through the pylorus into the second part of the duodenum by external pressure (using a lead glove for protection from radiation).

With the tube in position, so that the tip of the inner tube is in the second part of the duodenum, the patient is placed in a horizontal position on the fluoroscopy table. Four to $6 \mathrm{mg}$ oxyphenonium (Antrenyl, Ciba) is slowly injected intravenously, while $20 \mathrm{ml}$ of the above-mentioned barium mixture is slowly injected down the duodenal tube. The oxyphenonium soon produces a state of atonia in the duodenum and all movements then cease.

Oxygen, running at 4-6 $1 / \mathrm{min}$, is insufflated into the duodenum and a picture at maximum distension is taken. As soon as the film has been exposed the insufflation is stopped. This manoeuvre is repeated in the oblique positions and films again taken.

\section{Results}

The appearance of the duodenal loop on doublecontrast gas-distension duodenography is completely different from that on the routine barium examination (Fig. 1). Firstly, the pattern is constant from one film to the next and secondly the medial margin of the duodenal wall can be demonstrated in great detail. The medial margin of the second part of the duodenum is forced against the juxtaposed pancreas, allowing for detection of the earliest changes.

The duodenal mucosal folds, as shown by this method, run from margin to margin with no

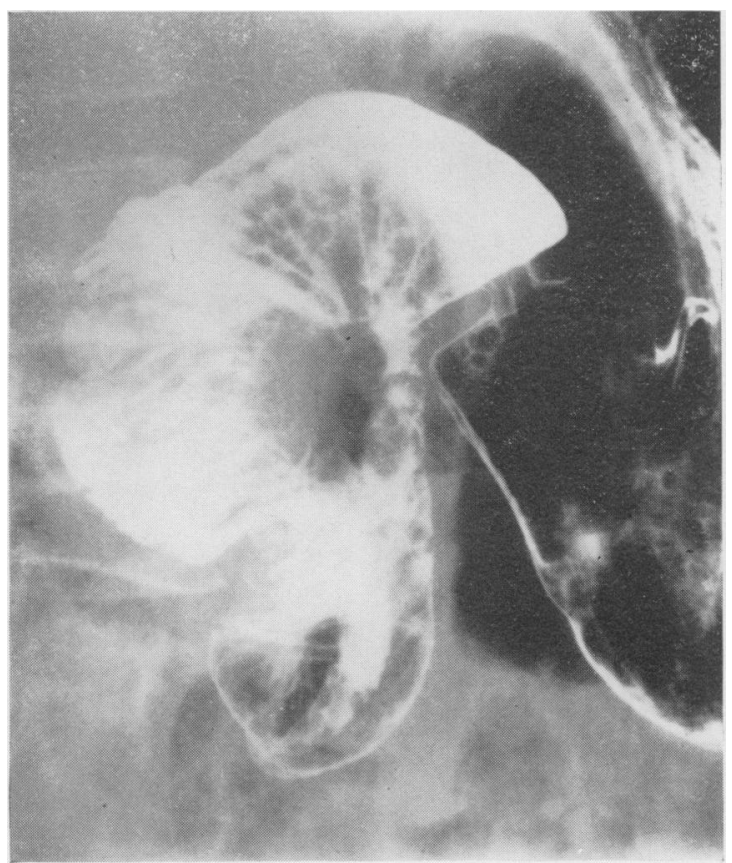

FIG. 3. Duodenography in a case of a carcinoma of the pancreas presenting with obstructive jaundice. The lesion was shown to be $2 \times 2.5 \times 3 \mathrm{~cm}$ in size. There is an extrinsic filling defect on the upper and medial margin of the second part of the duodenum associated with fine spiculation of the mucosal pattern.

vertical folds. Each outer fold has a corresponding inner fold. The distal half of the medial margin of the second part of the duodenum normally has a definite convexity.

In chronic pancreatitis there is loss of the medial aspects of the transverse folds. The medial margin tends to become straight with loss of the small convexities between folds (Fig. 2) and later with loss of the marked distal convexity of the inner margin. This inner margin also takes on small irregularities and in the late stages may even show an extrinsic filling defect similar to a carcinoma of the head of the pancreas. The famous inverted 3 sign of Frostberg may also be produced in the late stages of chronic pancreatitis.

The detection of carcinoma of the head of the pancreas can be increased from $25 \%$ in the conventional barium study to $75 \%$ by means of duodenography. It is therefore important to have a 'high index of suspicion.' One must certainly consider this condition in all cases of unexplained abdominal pain, especially if this radiates through to the back, and in all cases of obstructive jaundice in the absence of biliary stone formation. Localized loss of the medial margin of the transverse folds, localized straightening of the medial margin and a 
constant extrinsic filling defect (Fig. 3) indicate the presence of pancreatic pathology. These signs are visible on duodenography long before it is possible to detect them on the conventional barium meal. Later, large irregular filling defects on the medial margin, a pronounced Frostberg sign and marked irregularity of the duodenal margin supervene.

It may also be mentioned that double-contrast gas-distension duodenography has many other uses. In particular it is extremely valuable in showing the lining wall of diverticula, in the detection of ulcers or the rare neoplastic transformation that may occur. Lesions within the lumen of the duodenum, such as carcinoma of the ampulla of Vater, and constricting lesions such as the primary carcinoma of the duodenum, are particularly well demonstrated by this examination.

\section{Pre-operative percutaneous cholangiography}

The use of pre-operative percutaneous cholangiography has been one of the most important recent advances in the surgical management of obstructive jaundice. This is an accurate method of distinguishing between lesions within the liver substance

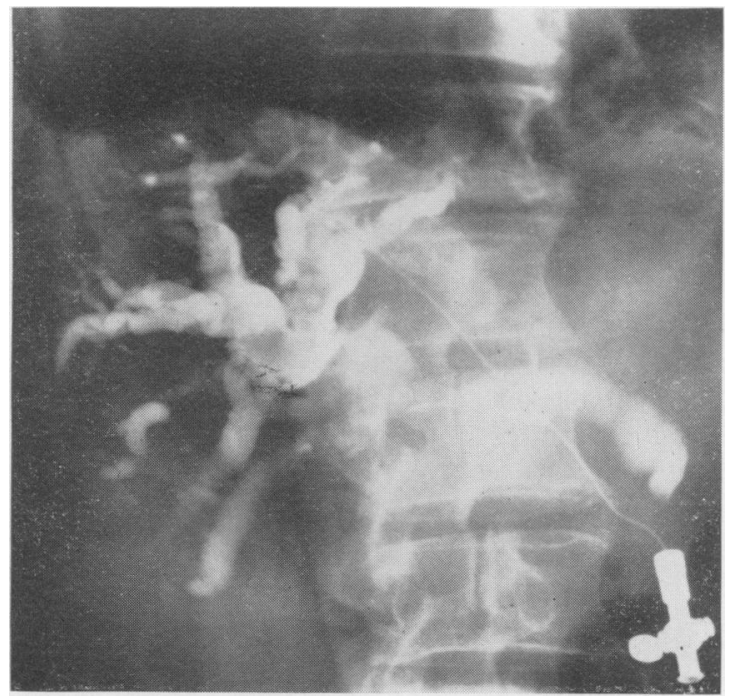

FIG. 4. Percutaneous cholangiography in a case of carcinoma of the pancreas causing obstructive jaundice. There are numerous filling defects inside the intrahepatic bile ducts caused by tumour extension into the liver. This was not visible to the surgeon who would have been unaware of this complication without radiographic visualization of the bile ducts.

which are hidden from the surgeon's view at laparotomy and those distal to the liver margin (Fig. 4). Furthermore, the radiographic appearance is frequently such as to make a diagnosis of the underlying pathology possible. Biliary stones, primary carcinoma, fibrotic strictures and carcinoma of the head of the pancreas are cases in point. The surgeon is thus able to plan his procedure with a knowledge of the site of obstruction, the size and disposition of the bile ducts and the underlying pathology. This results in a marked shortening of the operative time.

\section{Method}

It cannot be stressed too often that this is basically a pre-operative procedure and if used in this way it rarely leads to any untoward complications. This examination can be carried out under local or general anaesthetic. It must be performed under fluoroscopic control, and in this respect image intensification with television monitoring is of great assistance although by no means imperative.

Lengths of polyethylene tubing of $1.5 \mathrm{~mm}$ internal diameter (P.E.160) and approximately 20 $\mathrm{cm}$ long are prepared by flanging one end to fit a Seldinger tap adaptor. These are sterilized, preferably by $\gamma$-radiation, so as to be available at the time of the examination for their final preparation. Under sterile conditions, this tubing is threaded over a $12 \mathrm{~cm}$ long 18-gauge needle. The tubing is then firmly held between index and forefinger over the end of the needle and the free end of the tubing is drawn out with the aid of a pair of artery forceps. By pulling the polyethylene tubing out in this way it is narrowed to fit very snugly over the end of the needle. The tubing is then cut short and its end is drawn over the needle-tip to leave approximately $2 \mathrm{~mm}$ of bare needle for insertion into the liver.

The catheter and needle having been prepared, with the patient in the supine position the skin over the right hypochondrium is cleansed with chlorohexidine-alcohol solution. Where necessary, local anaesthetic is then injected and a small skin incision is made. A point just below the right costal margin along the lateral margin of the rectus abdominis is preferred. At this stage it is most important to open a track through the abdominal wall with a stout pair of artery forceps or sharp scissors. If a preformed track is not made in this way, the polyethylene tubing is caught in the abdominal wall and subsequently slides up the needle instead of adhering to it as the needle is inserted into the liver.

With the patient's breath held in inspiration, the needle and surrounding catheter are inserted in a slightly cranial and lateral direction up to the hilt of the needle. The needle is immediately removed, leaving the catheter behind buried in the liver substance. The tap fitting is screwed on and a syringe with saline is connected to it. While gently aspirating with the syringe, the catheter is slowly withdrawn until a free flow of bile is obtained. If 
blood is aspirated, the tubing is cleared and the withdrawal of the catheter with aspiration is then continued. It is permissible to reinsert the needle and catheter three times if no bile can be aspirated.

Once the bile has been aspirated the tap is closed and fixed onto the abdominal wall with strapping. Water-soluble contrast medium is used to outline the biliary system but before the injection starts one must be quite sure of clearing the system of air. Gas bubbles mimic stones, and with care this can be avoided. Twenty to $60 \mathrm{ml}$ of $45 \%$ Hypaque is used and then films are taken in the frontal plane, oblique positions and in the lateral position.

\section{Results}

It very occasionally happens that a dilated duct is not entered by this method, so that one cannot be absolutely sure that one is not dealing with a surgical obstructive jaundice in the event of a 'failed' examination. Usually, however, failure to enter a dilated bile duct indicates the presence of intrahepatic cholestasis and a collapsed biliary system.

In the presence of dilated bile ducts, the extent of the biliary dilatation and the site of the obstruction can be determined. Obstruction in the region of the right or left hepatic ducts or at their confluence invariably indicates a primary carcinoma of the bile ducts. This lesion is hidden from the surgeon's view and at laparotomy can easily be mistaken for intrahepatic cholestasis. In the context of the present review, this examination is particularly important in distinguishing between pancreatic lesions and calculous disease. Stones in the common bile duct show up as filling defects and the obstruction is often incomplete, with some contrast medium entering the duodenum. Carcinoma of the head of the pancreas often causes retrograde filling of an enlarged gall-bladder, but this sign cannot be relied upon as a cystic duct with a low implantation may also be obstructed by the neoplastic lesion, with consequent non-filling of the gall-bladder. The appearance at the obstructed end of the common bile duct is also of importance in distinguishing between stone formation and carcinoma. Where the obstruction is due to a stone, the contrast medium forms a half-moon shape at its end. In carcinoma of the pancreas this is often sharply cut off in a straight line or forms a beak-like projection, or may taper down to a point.

In the presence of obstructive jaundice, no patient should have a 'diagnostic' laparotomy without a pre-operative percutaneous cholangiogram. The procedure is reasonably free of complications and can be carried out with the simplest fluoroscopic equipment. It is incumbent on all radiologists to provide this service, just as it is imperative for all surgeons to demand it.

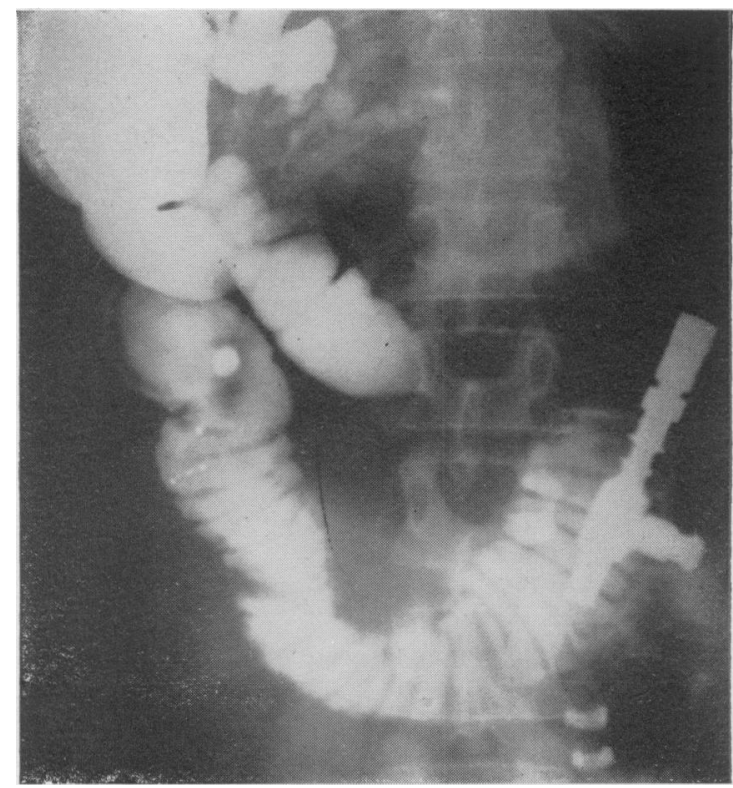

FrG. 5. Combined percutaneous cholangiography and duodenography in pancreatic carcinoma. The upper level of the tumour is shown by the site of the obstructed common bile duct while its lower level can be demonstrated by the irregular extrinsic filling defect seen at the junction of the second and third parts of the duodenum.

Having considered duodenography and percutaneous cholangiography, it may be mentioned that these two procedures may be combined (Fig. 5). This is particularly valuable in pancreatic carcinoma as it more accurately defines the extent of the lesion. The upper limit is shown by the obstructed common bile duct and the lower limit by the changes produced on the duodenal loop. Furthermore, the distorted anatomy will be shown, so that the surgeon will have an accurate idea of the relationships of the relevant organs.

Duodenography and percutaneous cholangiography, although a great advance on the conventional barium meal in the detection of pancreatic disease, are nevertheless indirect methods of investigation of the pancreas. In recent years, various direct methods of examination of the pancreas have been introduced. Some of these methods will now be considered.

\section{Direct pancreatography}

Gas in the form of air, oxygen and carbon dioxide has been used in radiology as a negative contrast agent for many years. Air encephalography, diagnostic pneumoperitoneum and perirenal air insufflation are all well known. It has, however, only recently been established that the pancreas can also be shown in this way. 


\section{Method}

Gas insufflation of the retroperitoneal tissue is carried out in the usual way. The trans-coccygeal method has the advantage that the needle is fixed and therefore danger of inadvertent intravenous gas injection is minimized. To show the pancreas, smaller quantities of gas must be used than in demonstrating the suprarenals: no more than $600 \mathrm{ml}$ are required. The patient should be moved from prone to supine and left to right lateral positions so that the gas can diffuse around the pancreas.

The stomach must be distended with gas. This is best achieved by administering a gas-producing tablet such as effervescent Redoxon. The gas-distended stomach tends to be immediately in front of the pancreas which is shown through it in the anteroposterior projection.

Tomography is required to actually delineate the pancreas. In the average size, 13,14 and $15 \mathrm{~cm}$ cuts in the supine position and cuts from $2 \mathrm{~cm}$ to the right of the midline to $9 \mathrm{~cm}$ to the left of the midline in the lateral projection will show the pancreas.

To enhance the visualization of the pancreas, secretin and water soluble contrast agents are used. It has been shown that secretin given in a dose of 1 unit/kg of body weight may increase pancreatic blood flow by a factor of up to 5 or 6 times. If a dose of contrast medium such as $60 \mathrm{ml} 45 \%$ Hypaque is given $30 \mathrm{~min}$ thereafter, it tends to produce a 'blush' of the pancreas. The obvious refinement of this procedure is in fact to combine this with selective coeliac axis arteriography or subselective arteriography. Both the arterial pattern and the 'blush' phase can then be obtained. This will be considered in some detail later.

Direct visualization of the pancreas by means of surrounding gas insufflation, following secretin and water-soluble contrast medium given either intravenously or by selective catheterization, is particularly valuable in demonstrating space-occupying lesions in the body and tail of the pancreas. By this means lesions such as carcinoma, insulinoma and cysts can be demonstrated.

\section{Pancreatic arteriography}

The arterial supply to the pancreas is derived from the coeliac axis and its branches and from the superior mesenteric artery (Fig. 6). It can be seen that the gastroduodenal artery, which is a major branch of the hepatic artery, divides into the anterior and posterior duodenal arcades which may rejoin to form the inferior pancreatic artery. The common inferior pancreatic artery usually arises from the superior mesenteric. The anterior and posterior duodenal arcades supply branches to the head of the pancreas and also frequently give a communicating branch to the transverse pancreatic artery.

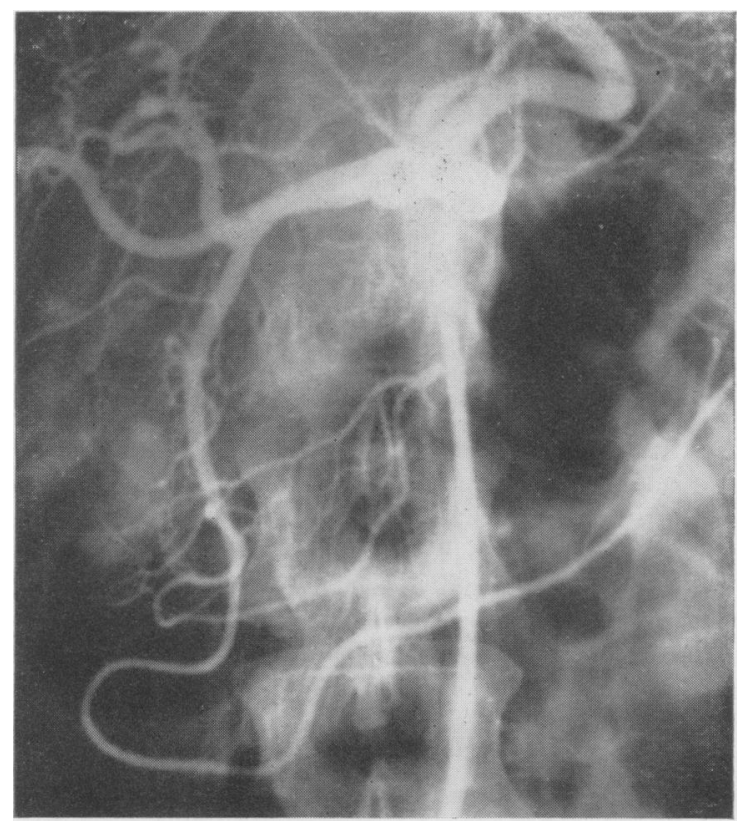

FIG. 6. Selective coeliac axis arteriography. The pancreatic arteries arise from the gastroduodenal artery which is a branch of the hepatic. These pancreatic arteries then join up to form the common inferior pancreatic artery which anastomoses with the superior mesenteric. The transverse pancreatic artery lies diagonally across the body of the first lumbar vertebra.

This artery is formed by branches from the splenic artery.

To achieve adequate visualization of these arteries, selective catheterization of the coeliac axis and superior mesenteric arteries is required. This is done by the use of a preformed radio-opaque catheter, under fluoroscopic control. In this respect image intensification with television monitoring is of great assistance. The femoral route is the most convenient, and by the use of suitably placed skin markers this can be achieved with rapidity in the vast majority of cases.

The Swedish workers recommend simultaneous coeliac axis and superior mesenteric catheterization. Subselective catheterization promises to give better results and requires only one arterial puncture. This is performed by passing a suitably curved teflon catheter up the Grey-Odman catheter which has been placed in position in the coeliac axis or mesenteric artery. By passing this smaller catheter well into the hepatic artery or into the common inferior gastro-duodenal artery, a complete demonstration of the pancreatic vessels is possible with a small amount of contrast medium (10-15 ml Conray '420').

Arteriography has been considered a valuable means for the diagnosis of malignant lesions in 
internal organs such as the brain, kidney and liver, and in bone disease. This is so largely because in these organs vascular displacement and a pathological 'malignant' circulation with pooling of contrast medium, arteriovenous shunting and multiple tortuous arterioles which do not taper are seen. 'Tumour staining' by the contrast medium may also be present. However, in pancreatic carcinoma these signs are hardly ever present or only minimal. Thus the value of angiography in this condition has been largely ignored. In recent years attention has been drawn to the less obvious but equally valid angiographic signs which can be detected in cases of pancreatic carcinoma. Arterial deformity due to infiltration of the arterial wall produces appearances of marked irregularity of the margins, narrowing of the calibre and irregular tortuosity of short segments of an artery. These signs may be present in the large arteries adjacent to the pancreas, such as the splenic and gastroduodenal arteries, as well as in the pancreatic arterial branches. There may also be the sudden truncation of an artery due to thrombosis. This can occur in both chronic inflammatory disease and in malignant infiltration.

Pancreatic arteriography requires a special mention in considering the diagnosis of insulinoma. Although this is a rare tumour, its removal can lead to a complete cure of severe attacks of hypoglycaemia. This condition can now be accurately diagnosed by biochemical means. Surgery can be difficult as there may be multiple adenomata or the tumour may be deeply imbedded in the pancreatic tissue. These adenomata may, however, be shown by arteriography and this will be of great assistance to the surgeon. One further point in connection with coeliac axis arteriography may be mentioned. Occasionally in pancreatic carcinoma or chronic pancreatitis there may be portal vein obstruction. By taking films at 10-14 sec after the arterial injection the splenic and portal veins can be visualized. It can then be shown whether the portal vein is patent, and if so whether it is normal in calibre, and it is also possible to demonstrate the presence of collateral venous filling by this means.

Direct pancreatography can thus be carried out by retroperitoneal gas insufflation and enhancement of pancreatic tissue density by the use of secretin and intravenous water-soluble contrast medium, as well as by the method of selective or subselective pancreatic angiography. It is more than likely that in the near future these procedures will be combined. Retroperitoneal gas insufflation will be induced, the coeliac axis will be catheterized, secretin given and then angiotomography in the antero-posterior and lateral planes will be carried out with a multi-layer cassette. The immediate films will show the arterial phase and the late films will

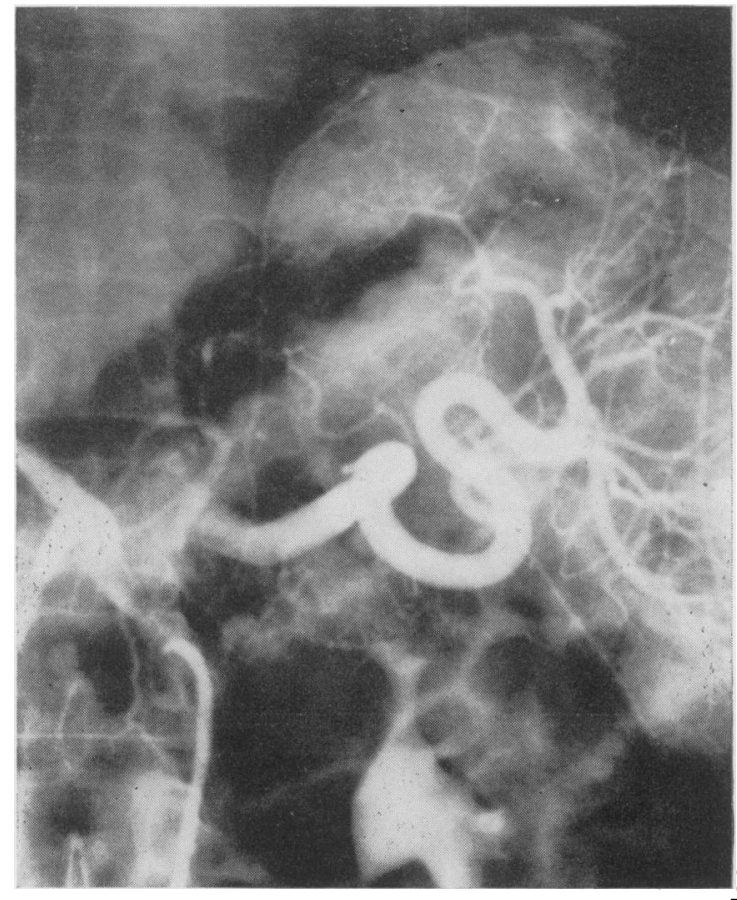

FIG. 7. Selective coeliac axis arteriography showing dilated and tortuous pancreatic arteries arising from the splenic artery in a case of chronic pancreatitis. The late venous phase showed a marked collateral circulation with non-visualization of the portal vein indicating portal venous obstruction.

demonstrate the tissue 'blush' phase. This procedure will, of course, necessitate having a tomographic unit in the angiography room. At present this is an extremely rare occurrence. Angiotomography by and large requires, at present, moving the patient a considerable distance with the selective catheter in situ, and transference from radiographic table to trolley and back to a radiographic table again. The angiotomographic unit would be the practical solution for undertaking this type of examination.

\section{Duct pancreatography}

Direct operative cannulation of the pancreatic duct, drainage and then radiographic visualization can be carried out without complications provided the contrast medium is not forcibly injected but allowed to run in by minimal hydrostatic pressure. It is the forcible injection of water-soluble contrast medium into the pancreatic duct which leads to chemical pancreatitis. At present, operative pancreatography is only used in chronic pancreatitis, where it enables the surgeon to locate the site of duct obstruction and the state of the ducts proximal to it. A great measure of success in the relief of pain due to chronic pancreatitis can be achieved if 
free drainage can be re-established. Where the obstruction is near the ampulla of Vater it can be relieved by a direct approach. However, if the obstruction is in the body and tail it may produce the 'chain of lakes' appearance which will require jejuno-pancreostomy for relief of symptoms. It would thus appear that at present the rational approach by the surgeon to chronic pancreatitis would entail the use of operative pancreatography.

A very recent contribution has shown that the peroral cannulation of the ampulla of Vater and the subsequent visualization of the common bile duct and pancreatic duct is a distinct possibility. If a method such as this becomes a practical procedure it will immediately make a considerable contribution to the pre-operative diagnosis and to the management of pancreatic disease.

\section{Pancreatic photo-scanning with ${ }^{75}$ selenium- methionine}

Photo-scanning is a technique which is being used with increasing frequency in the diagnosis of intracranial lesions and in thyroid, lung, liver and renal diseases. At present the use of this diagnostic method is limited by the availability of suitable equipment, but there is no doubt that in the near future the necessary apparatus will become more widely distributed. Recently a method for the visualization of the pancreas has been devised. This is based on the fact that between $5 \%$ and $10 \%$ of ingested amino acids appear in the pancreas within $2 \mathrm{hr}$. These amino acids are the substrate for the pancreatic synthesis of digestive enzymes. This applies to the sulphur-containing amino acid methionine as well. The substitution of 75 selenium for the sulphur in the methionine molecules produces an analog with all the physiological properties of methionine. This is thus a suitably labelled pancreasspecific compound which can be employed in photoscanning for pancreatic diseases.

${ }^{75}$ Selenium-methionine (Seleno-methionine) if given intravenously in a dose of $3 \mu \mathrm{Ci} / \mathrm{kg}$ body weight will visualize the pancreas on photo-scanning starting $\frac{1}{2} \mathrm{hr}$ after the injection. This dose amounts to approximately $200 \mu \mathrm{Ci}$ in the average sized person. With a physical half-life of 120 days and an effective biological half-life of 56 days, this amounts to $0.6 \mathrm{rad}$ total body radiation. The pancreas retains $7 \%$ of the seleno-methionine given for $4 \mathrm{hr}$, while the liver retains $10 \%$ for 1 day. The photo-scan thus shows not only the pancreas but also the liver. It is thus obvious that in the presence of hepatic enlargement the liver scan will overlap that of the pancreas. As both the liver and pancreas are visualized by this technique it is usually necessary to scan the liver alone using colloidal radio-gold $\left({ }^{198} \mathrm{Au}\right)$. This allows overlaps of the pancreas by the liver edge to be identified when necessary.

Various methods have been used in the attempt to facilitate the concentration of the radio-nuclide in the pancreas. These include a high protein diet, the exhibition of pancreatic enzymes such as secretin and pancreatozymin, the use of morphine and more recently the use of whisky. However, it is yet to be demonstrated that any of these measures is more satisfactory than the next, with the possible exception of the high protein feed. Initially this procedure entails no more than having an intravenous injection and lying quietly in the supine position for about $1 \mathrm{hr}$ while the photo-scan is being performed. The simplicity of this technique compared with that of the other methods of direct visualization of the pancreas is its greatest advantage.

\section{Method}

The patient has a normal evening meal and also a high protein feed at midnight. An early breakfast of two glasses of milk, one slice of toast and a cup of coffee without sugar is then given. The dose of intravenous seleno-methionine $(3 \mu \mathrm{Ci} / \mathrm{kg}$ body weight) is administered. Half an hour later photoscanning anteriorly with the subject lying supine is commenced and lasts approximately $45 \mathrm{~min}$. The 5 in. probe with the $5 \mathrm{in}$. broad focussing collimator appears to give the best photo-scans.

\section{Results}

As has been mentioned previously, both the liver and pancreas are visualized by performing the photo-scan after giving seleno-methionine. Normally there is a well-demarcated gap between the scan of the liver and that of the head of the pancreas. The shape of the pancreas has been described as being of three types, the high transverse, the horseshoe and the sigmoid. The size, shape and position of the pancreas can thus be demonstrated in the majority of cases (Fig. 8). However, even in the normal subject there may be no visualization of the pancreas on the photo-scan. The reason for this is, at present, not apparent, but this does mean that non-visualization cannot be accepted as a pathognomonic sign of pancreatic disease. This is, however, commonly present in chronic pancreatitis.

The signs that do indicate the presence of a pancreatic lesion on the photo-scan include a localized filling defect and displacement of the normal pancreatic tissue as well as distortion and narrowing of any part of the pancreatic scan. An associated filling defect in the liver may also be demonstrated, indicating the presence of liver metastasis.

No doubt further refinements in the technique of photo-scanning will make this a more accurate diagnostic tool in the detection and localization of 


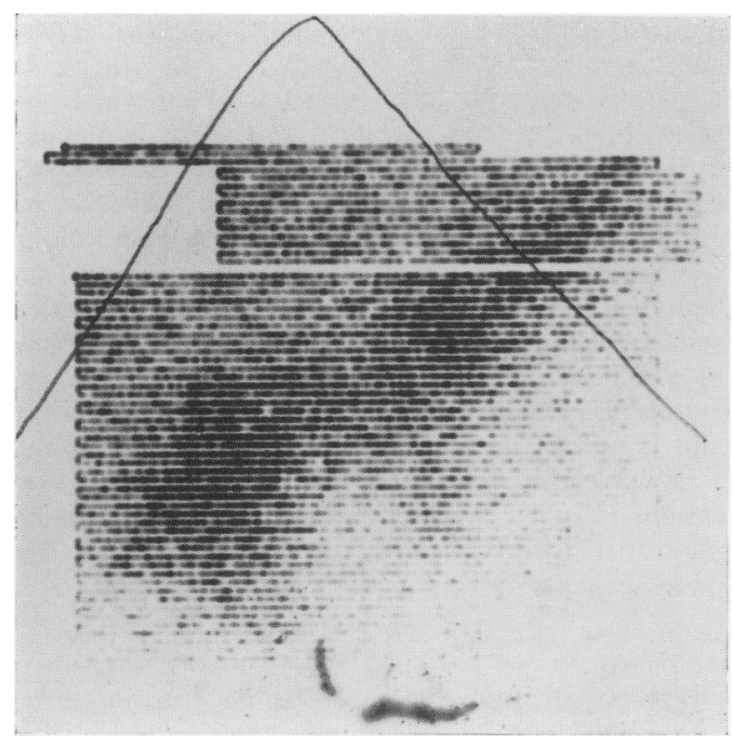

FIG. 8. A photo-scan of the normal pancreas of the high transverse type.

pancreatic disease, but first and foremost many more centres must acquire the apparatus to enlarge the sum total of experience with scanning techniques. The benefits and limitations of this apparatus and the various methods employed will then be demonstrated.

\section{Conclusion}

The large number and wide variety of methods required to demonstrate the pancreas reveals, in fact, that the ideal method does not exist at present. Theoretically the ideal method would be an excretion pancreaticogram similar to the intravenous pyelogram. This would show the parenchyma in the early stages and the duct patterns and major duct in the later stages. Such an examination may only become available in the distant future but may indeed be just around the corner. Until this ideal 'excretory pancreaticogram' arrives on the diagnostic scene we must make do with the techniques at present available, proceeding from the least complex and time-consuming examinations to the more complicated procedures. Allowances must, of course, be made for any particular problem in one patient and the apparatus available to carry out any particular examination. However, the first essential is that clinicians and radiologists should focus their attention on the pancreas in all cases of unexplained upper abdominal pain and noncalculous obstructive jaundice until such time as the utopian 'excretory pancreaticogram' becomes a routine diagnostic procedure.

\section{Acknowledgments}

I wish to thank Mr Gilson of the Photographic Department, Royal Free Hospital, and his staff who prepared the illustrations. I also wish to thank Dr S. Raia for Fig. 5 and Dr R. Melmed for Fig. 8. Dr R. Melmed also read and corrected the section dealing with photo-scanning of the pancreas.

\section{Bibliography}

Conventional radiological examination of the pancreas

KeMP HARPER, R.A. (1953) Modern Trends in Diagnostic Radiology (Ed. by J. W. McLaren), 2nd series, p. 218. Butterworth, London.

KEMP HARPER R.A. (1958) A Text-Book of X-Ray Diagnosis (Ed. by S. Cochrane-Shanks and P. Kerley), p.530. Lewis, London.

\section{Duodenography}

JACQuemet, P., Liotta, D. \& Mallet-Guy, P. (1965) The Early Radiological Diagnosis of Diseases of the Pancreas and Ampulla of Vater. Charles C. Thomas, Illinois.

Mallet-Guy, P. \& Jacquemet, P. (1963) Resultats de 500 cas de duodenenographie hypotonique. J. Radiol. Electrol. 44, 249.

RAIA, S. (1967) Simultaneous percutaneous cholangiography and duodenography. Surgery, (In press).

RaIA, S. \& KreEL, L. (1966) Double contrast gas distension duodenography using the Scott-Harden gastro-duodenal tube. Gut, 7, 420 .

Operative pancreatography

Pollock, A.V. (1958) Pancreatography in diagnosis of chronic relapsing pancreatitis. Surg. Gynec. Obstet. 105 706.

EgDAHL, R.H. \& Hume, D.M. (1963) Surgery in pancreatitis. Amer. J. Surg. 106, 471.

BARTLETT, M.K. (1965) Treatment of recurrent pancreatitis transduodenal sphincteroplasty and experience with pancreatic inhibition and retrograde drainage after resection. Amer. Surg. 31, 773.

Gas insufflation pancreatography

(Secretin + water soluble contrast medium intravenously)

Kisseler, B., Leistner, G.H. \& Barth, E. (1964) A new method for the roentgenologic opacification of the pancreas. Radiology, 83, 6 .

Pancreatic angiography

Evans, J.A. (1965) Techniques in the detection and diagnosis of malignant lesions of the liver, spleen and pancreas. Radiol. Clin. N. Amer. 3, 567.

Kreel, L. \& Williams, R. (1964) Arteriovenography of the portal system. Brit. med. J. ii, 1500.

LuNDERQuisT, A. (1965) Angiography in carcinoma of the pancreas. Acta radiol. (Stockh.), Suppl., 235.

Paul, R.E., Miller, H.H., Khan, P.C., Callow, A.D., Edwards, T.L., Jr. \& PATTERSON, J.R. (1965) Pancreatic angiography with application of subselective angiography of the coeliac or superior mesenteric artery to the diagnosis of carcinoma of the pancreas. New Engl.J. Med. 272, 283.

Pre-operative percutaneous cholangiography

Atkinson, M., HAPPey, M.G. \& SMiddy, F.G. (1960) Percutaneous transhepatic cholangiography. Gut, 1, 357.

Burckhard, H. \& Muller, W. (1921) Versuche uber die Punktion der Gallenblase und ihre Rontgendarstellung. Dtsch. Z. Chir. 162, 168.

Huard, P. \& Do-XuaN, Hop (1937) La ponction transhepatique des canaux biliaries. Bull. Soc. med.-Chir. Indoch. 15, 1090. 
Remolar, J., Katz, S., Rybak, B. \& Pellizari, O. (1956) Percutaneous transhepatic cholangiography. Gastroenterology, 31, 39.

Shaldon, S. (1962) The investigation of bile ducts by percutaneous cholangiography. Proc. roy. Soc. Med. 55, 587.

Sobredo, E.E. \& Garcia Siguerdo, P.R. (1954) Colangiografia transabdominal. Orientacion med. 3, 1585.

Peroral cannulation of the ampulla of Vater for direct cholangiography and pancreatography

Rabinov, K.R. \& Simon, M. (1965) Peroral cannulation of the ampulla of Vater for direct cholangiography and pancreatography - preliminary report of a new method. Radiology, 85, 693.
Pancreatic photo-scanning with seleno-methionine (75Se)

BlAU, M. \& BENDER, M.A. (1962) SE75-selenomethionine for visualisation of pancreas by isotope scanning. Radiology, 78, 974.

Blau, M. \& Manske, R.F. (1961) Pancreas specificity of SE75-selenomethionine. J. nucl. Med. 2, 102.

Haynie, T.P., Svoboda, A.L. \& Zuidema, G.D. (1964) Diagnosis of pancreatic disease by photo-scanning. J. nucl. Med. 5, 90.

King, E.R., Sharpe, A., Grubb, W., Brock, J.S. \& GreenberG, L. (1966) A study of the morphology of the normal pancreas using SE75-methionine photo scanning. Amer. J. Roentgenol. 96, 659.

Rodriguez-Antunez, A. (1964) Pancreatic scanning with selenium-methionine, utilising morphine to enhance contrast. Cleveland Clin. Quart. 31, 213. 